\title{
Améloblastome historique de la mandibule
}

\author{
Maryame Abou-Elfadl*, Loubna Elhani, Mohammed Roubal, Mohammed Mahtar, Fatmi Kadiri \\ Service d'ORL et de chirurgie cervico-faciale, hôpital 20 Août, Casablanca, Maroc \\ dr_abouelfadl_m@hotmail.fr
}

(Reçu le 19 septembre 2014, accepté le 4 janvier 2015)

Mots clés :

améloblastome géant / mandibule / exérèse /récidive

Key words:

giant ameloblastoma / mandible / enucleation / recurrence
Résumé - Introduction : L'améloblastome est une tumeur bénigne odontogène, caractérisée par son agressivité locale, atteignant le plus souvent la mandibule. Le diagnostic est généralement fait devant l'apparition d'une tuméfaction mandibulaire. Son traitement nécessitait l'exérèse chirurgicale et une surveillance régulière en raison du risque de récidive. Nous rapportons un cas historique d'améloblastome mandibulaire géant, diagnostiqué à un stade tardif, compliquant la prise en charge et la reconstruction. Observation : Il s'agissait d'une patiente âgée de 50 ans, se présentant pour un améloblastome mandibulaire géant, diagnostiqué à un stade avancé du fait de la consultation tardive, et ayant bénéficié d'une exérèse complète avec reconstruction. Discussion-Conclusion : Les formes géantes de l'améloblastome constituent un problème thérapeutique majeur car elles nécessitent une résection complète de la mandibule engageant le pronostic fonctionnel et esthétique. La présentation de ce cas géant d'améloblastome nous rappelle les difficultés d'accès aux soins et le suivi des malades dans les pays en voie de développement.

\begin{abstract}
Historical ameloblastoma of the mandible. Introduction: Ameloblastoma is a benign odontogenic tumor characterized by its local aggressiveness, reaching the mandible most often. The diagnosis is usually made before the occurrence of a mandibular swelling. Its treatment requires surgical excision and regular monitoring because of the risk of recurrence. We report a case history of a giant mandibular ameloblastoma diagnosed at a late stage, complicating the management and reconstruction. Observation: This was a 50-year-old female patient consulting for a giant mandibular ameloblastoma diagnosed late because of the advanced stage consultation, and having received a complete resection with reconstruction. Discussion-Conclusion: A giant form of ameloblastoma is a major therapeutic problem, as they require complete resection of the mandible, engaging the functional and aesthetic prognosis. The presentation of this giant case of ameloblastoma reminds us of the difficulties of access to care and monitoring for patients in developing countries.
\end{abstract}

\section{Introduction}

L'améloblastome est une tumeur odontogène, qui dérive des cellules de l'émail dentaire, atteignant le plus souvent la mandibule. Bien qu'elle soit bénigne, elle est localement agressive et demande le plus souvent une résection chirurgicale large.

Le diagnostic est généralement fait à l'occasion de manifestations dentaires ou devant l'apparition d'une tuméfaction mandibulaire. Les auteurs rapportent un cas historique d'améloblastome mandibulaire géant, diagnostiqué à un stade avancé du fait de la consultation tardive compliquant la prise en charge et la reconstruction, ayant bénéficié d'une exérèse complète.

\section{Observation}

Il s'agissait d'une patiente âgée de 50 ans, sans antécédent pathologique. Elle consultait pour une énorme masse de la région mandibulaire évoluant depuis huit ans, qui augmentait progressivement de volume, sans douleur, sans trouble de déglutition, ni dyspnée, dans un contexte de conservation de l'état général.

L'examen clinique de la face montrait une énorme masse aux dépens de la région mandibulaire droite et gauche mesurant $200 \times 120 \times 130 \mathrm{~mm}$, fixe, de consistance dure par endroits, rénitente par d'autres, non douloureuse, le revêtement cutané 


\section{A S C L I N I Q U E}

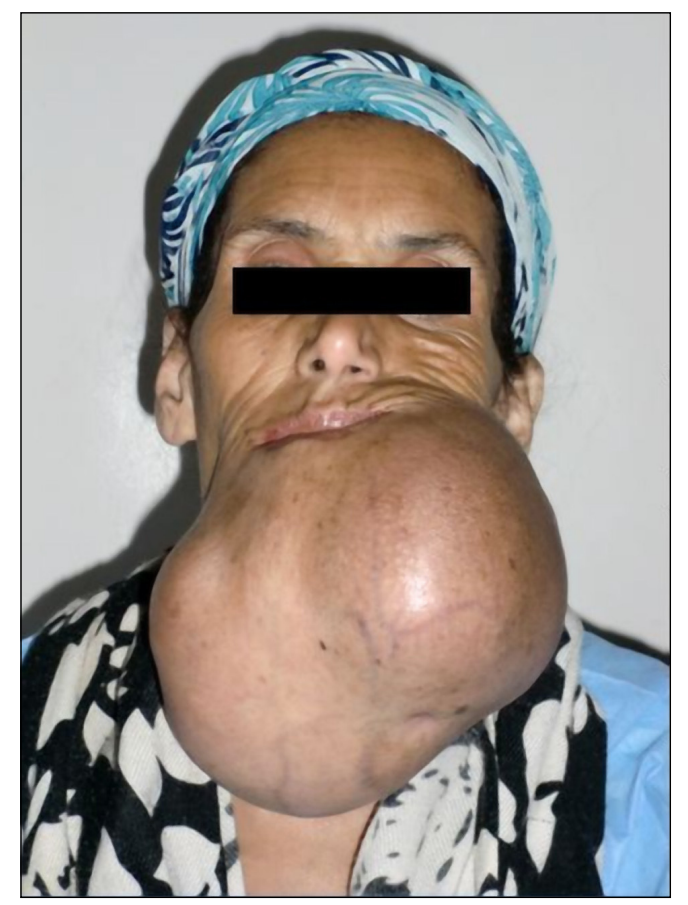

Fig. 1. Examen de face permettant de visualiser l'ampleur de la tuméfaction mandibulaire.

Fig. 1. Facial view underlying the extent of the mandibular swelling.

en regard étant normal (Fig. 1). L'examen cervical n'a pas trouvé d'adénopathie.

L'examen de la cavité buccale objectivait une masse étendue sur toute l'hémi-mandibule gauche, la moitié de l'hémi-mandibule droite jusqu'à la seconde prémolaire. Cette tuméfaction bombait le plancher buccal et refoulait la langue en arrière et à droite.

Le scanner cervico-facial a retrouvé une volumineuse tumeur mandibulaire mesurant $165 \times 140 \mathrm{~mm}$, latéralisée à gauche, multilobulée, soufflant les corticales osseuses, siège de zones charnues rehaussées après injection de produit de contraste. Elle arrivait au contact du processus alvéolaire du maxillaire gauche qu'elle envahit.

En bas, elle arrivait au contact de l'os hyoïde et du larynx qui étaient en place. Elle refoulait en arrière la trachée et la tyroïde, sans infiltration des parties molles et des espaces profonds de la face, sans adénopathie cervicale visible. Les articulations temporo-mandibulaires étaient respectées (Fig. 2).

Malgré sa taille géante, la tumeur a été opérable, du fait de ses limites nettes et du fait qu'elle n'envahissait pas les parties molles de voisinage ni les espaces profonds de la face.

La patiente a bénéficié d'un traitement radical avec une mandibulectomie et une mise en place d'une plaque de reconstruction mandibulaire. Les suites opératoires ont été simples et une réintervention pour reconstruction a été programmée ultérieurement (Fig. 3).

L'examen anatomopathologique a conclu à un améloblastome de type folliculaire sans caractère de malignité.

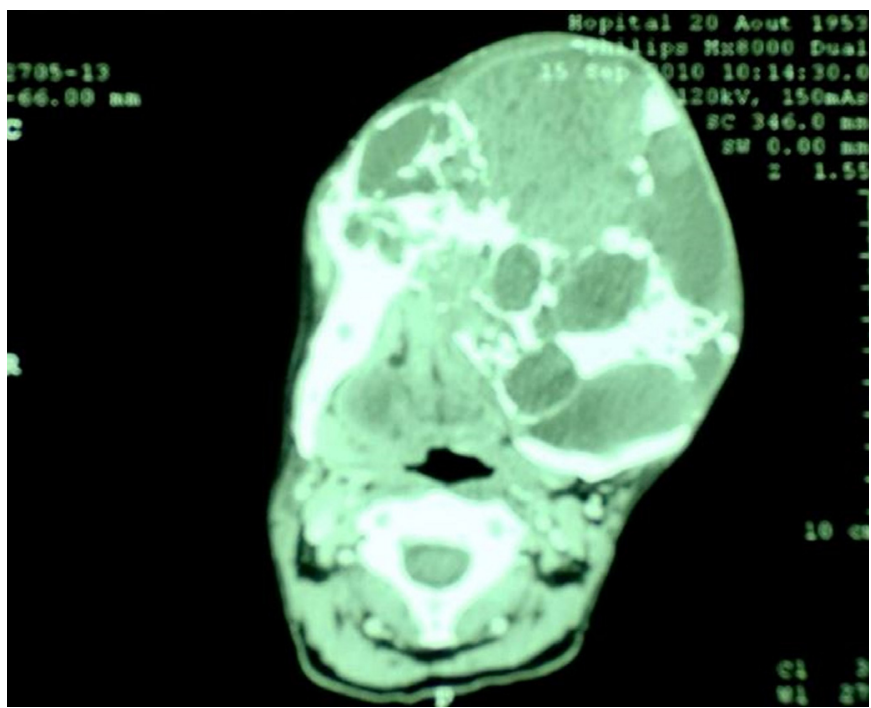

Fig. 2. Examen scannographique permettant le bilan d'extension de l'améloblastome.

Fig. 2. CT allowing the assessment of the ameloblastoma extent.

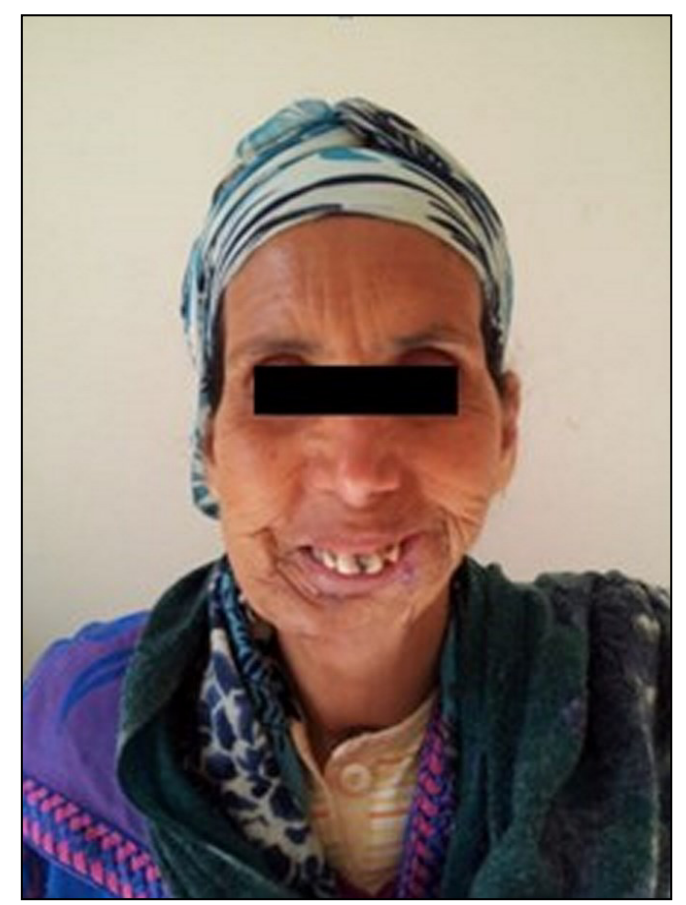

Fig. 3. Aspect de face, un an après l'exérèse de l'améloblastome. Fig. 3. Facial aspect, one year after the surgical enucleation of the ameloblastoma.

\section{Discussion}

L'améloblastome est une tumeur odontogène bénigne, mais qui nécessite un diagnostic précoce du fait de son caractère 
invasif. Elle touche principalement les personnes entre 30 et 60 ans, sans prédilection de sexe [1].

Elle représente 11 à $18 \%$ de toutes les tumeurs odontogéniques et représente la deuxième tumeur odontogène la plus fréquente après les odontomes [1]. La mandibule est atteinte dans $80 \%$ des cas (angle : $70 \%$, région prémolaire : $20 \%$, incisive : $10 \%$ ) [2].

La découverte de l'améloblastome est le plus souvent fortuite car peu symptomatique et indolore dans la grande majorité des cas. Les circonstances de découverte sont plus dominées par les déformations faciales et/ou les pertes dentaires.

Les images radiologiques de l'améloblastome sont diverses. L'image polygéodique en « nids d'abeilles » est la plus fréquente, traduisant une destruction osseuse polygéodique soufflant la corticale osseuse [2].

Selon l'organisation mondiale de la santé, plusieurs formes d'améloblastomes existent : type solide/multikystique, type extraosseux/périphérique, type desmoplastique et type monokystique. Selon l'histologie, on note le type folliculaire, plexiforme et plus rarement la forme à cellules granuleuses [3]. Les améloblastomes sont à différencier des myxomes odontogènes, des kératokystes odontogéniques, des fibromes améloblastiques et des granulomes centraux à cellules géantes. Leur présentation clinico-radiologique est pratiquement similaire. Seule l'histologie permet de les différencier [2].

L'évolution métastatique de l'améloblastome est rare mais possible, plusieurs cas ont été rapportés dans la littérature [4]. Il n'y a pas de consensus sur les modalités thérapeutiques. Deux techniques chirurgicales s'opposent. Le traitement conservateur consiste en une énucléation-curetage de la tumeur avec maintien du périoste pour la régénération osseuse, en particulier chez l'enfant $[1,5]$. Il semble indiqué en cas de petite tumeur unikystique, bien limitée, avec des tables osseuses non rompues [1,5]. Le traitement radical consiste en une exérèse large avec marges saines de $2 \mathrm{~cm}$. Il semble indiqué dans les tumeurs diffuses, mal limitées, polykystiques [1]. Le risque de récidive serait de l'ordre de 36 à $100 \%$ après traitement conservateur et de l'ordre de 0 à $21 \%$ après traitement radical [5]. Les partisans de l'approche conservatrice comme c'est mentionné dans la référence [5] estiment que l'améloblastome, malgré l'agression locale, est une tumeur bénigne. À l'inverse, les partisans de l'approche radicale mentionnent que le curetage et l'énucléation engendrent plus de récidive avec des taux entre 55 et $90 \%$ [5]. Ils recommandent une résection radicale avec une marge d'au moins $2 \mathrm{~cm}$, ce qui peut emporter la moitié ou toute la mandibule $[1,5]$.

Il est préférable d'envisager la reconstruction mandibulaire dans le même temps que l'exérèse chirurgicale de l'améloblastome. En effet, les reconstructions secondaires sont toujours plus difficiles. Si la technique de reconstruction de référence est le lambeau libre de fibula, les équipes n'ont pas toutes accès aux techniques microchirurgicales. En cas d'utilisation de plaque de reconstruction, il est souhaitable de couvrir celle-ci avec un lambeau musculaire type grand pectoral sous peine de voir progressivement la plaque s'extérioriser à la peau [1].

\section{Conclusion}

L'améloblastome apparaît comme une tumeur localement envahissante, à haut risque de récidive, ce qui justifie la nécessité d'un diagnostic précoce et d'un traitement adapté.

La présentation de ce cas d'améloblastome géant nous évoque les difficultés d'accès aux soins et le suivi des malades dans les pays en voie de développement, ce qui complique leur prise en charge.

\section{Conflits d'intérêt : aucun}

\section{Références}

1. Sharma A, Shaikh AM, Deshmukh SV, Dabholkar JP. Radical management of giant ameloblastomas: A Case Series. Indian J Otolaryngol Head Neck Surg 2012;64(4):399-401.

2. More C, Tailor M, Patel HJ, Asrani M, Thakkar K, Adalja C. Radiographic analysis of ameloblastoma: A retrospective study. Indian J Dent Res 2012;23(5):698.

3. Hunasgi S, Koneru A, Chauhan DS, Guruprasad Y. Rare Giant Granular Cell Ameloblastoma: A Case Report and an Immunohistochemical Study. Case Reports in Dentistry 2013; Article ID 372781.

4. Van Dam SD, Unni KK, Keller EE. Metastasizing (malignant) ameloblastoma: Review of a unique histopathologic entity and report of mayo clinic experience. J Oral Maxillofac Surg 2010; 68(12):2962-74.

5. Adeyemo WL, Bamgbose B0, Ladeinde AL, Ogunlewe MO. Surgical management of ameloblastomas: conservative or radical approach? A critical review of the literature. Oral Surgery. 2008; 1(1):22-7. 